\title{
PROTECCIÓN OCULAR: IMPORTANCIA Y USO
}

\author{
EYE PROTECTION: IMPORTANCE AND USE
}

CRISTINA SERRANO RAMOS(1), LOURDES JIMÉNEZ BAJO(1), MARÍA JEREZ FIDALGO ${ }^{(2)}$,
SILVIA O'CONNOR PÉREZ ${ }^{(1)}$, IGNACIO BARDÓN FERNÁNDEZ-PACHECO $^{(1)}$, COVADONGA CASO PITA $^{(1)}$,

(1) Servicio de Prevención. Hospital Clínico San Carlos. Madrid. España.

(2) Servicio de Oftalmología. Hospital Clínico San Carlos. Madrid. España.

\section{RESUMEN}

La traumatología ocular supone el $10 \%$ de la traumatología general en España, siendo los accidentes oftalmológicos de origen laboral en su mayoría. Nuestro objetivo es conocer la prevalencia y etiología en nuestro medio, proponiendo medidas preventivas para disminuirlos en número y gravedad.

Realizamos un estudio descriptivo trasversal en 41 trabajadores atendidos en un Servicio de Urgencias Oftalmológicas en un Hospital del Servicio Público de Salud de la Comunidad de Madrid durante el mes de Abril de 2006. Mediante cuestionario específico analizamos las siguientes variables: edad sexo, profesión, sector, dispensación y uso de protección ocular, reincidencia, diagnóstico y asistencia médica

Los resultado obtenidos muestran que un 25.6\% de accidentes oculares son de origen laboral, que se dan en el $87 \%$ en varones, con una edad media de 35 años; pertenecen al sector de la construcción un $85.4 \%$, que maneja equipo de soldadura o radial. El diagnóstico más frecuente es el impacto de cuerpo extraño y se trataba de accidente reiterado en el $32 \%$ de los casos. No posee protección ocular el $51.2 \%$ y de los que la poseen no la usa el $48 \%$. Por todo ello nuestro esfuerzo debe encaminarse a la información y formación sobre la protección ocular, en trabajador y empresario

El hecho de que el $46 \%$ podría haber sido tratado de forma ambulatoria supone que médico del trabajo debe estar entrenado en el manejo de la traumatología ocular por su frecuencia y la necesidad de tratamiento inmediato en el lugar de trabajo.

Palabras clave: Accidentes laborales oftalmológicos, protección ocular, información-formación.

(Med Segur Trab 2008; 54 (213):81-86)

\begin{abstract}
Ten percent of the overall traumas in Spain are in the eye, these accidents where mostly at work. The aim of our study is to determine the prevalence and etiology in our area and propose preventive measures to decrease the number of accidents and their severity.
\end{abstract}

We conducted a descriptive study in 41 workers attended in an eye emergency department (Hospital San CarlosApril2006) with a specific questionnaire (variables: age, sex, occupation, industry, supply and use of eye protection, recidivism, diagnosis and medical care).

The results showed $25.6 \%$ of industrial eye accidents, $87.2 \%$ where in males with an average age of 35.15 years, $85.4 \%$ belong to the construction sector. The most frequent diagnosis was the impact of foreign body. We realize that $51.2 \%$ do not have safety glasses, and between those who have it, $48 \%$ do not wear it. So, our efforts must be geared to information and training on eye protection, worker and employer.

The $46.3 \%$ of accidents only need ambulatory treatment, this implies that occupational medical should be trained in handling of eye injuries by their frequency and need for immediate treatment in the workplace.

Key words: Eye injuries, safety glasses.

(Med Segur Trab 2008; 54 (213):81-86)

\footnotetext{
Correspondencia:

Dra. Cristina Serrano Ramos

Hospital Clínico San Carlos

Profesor Martín Lagos S/N

28040 Madrid. España

cserranor.hcsc@salud.madrid.org

Teléfono: 678412333
} 


\section{INTRODUCCIÓN}

En España la patología traumatológica ocular supone la tercera causa de ceguera o secuelas importantes, detrás de la retinopatía diabética y del glaucoma por orden de frecuencia. Los accidentes oculares, a pesar de la pequeña superficie corporal que ocupan los ojos, suponen alrededor del 10\% de todos los accidentes del organismo, siendo de etiología laboral gran parte de los mismos. (1,2) Según el Grupo para el Estudio Multicéntrico de los Traumatismos Oculares en España (GEMTO), los accidentes laborales suponen la primera causa de traumatismo ocular, representando el $23,2 \%$ del total. Predominan en hombres $(26,7: 1)$ de entre 20 y 40 años (60\%), población joven y activa, por lo que suponen un problema socioeconómico. El sector industrial supone un $40 \%$ y la construcción un $32,5 \%$ del total de los accidentes oculares. Con estos datos podemos decir que los accidentes oftalmológicos laborales son una parte muy importante de la patología laboral, y esto ocurre tanto en España como en otros países. $(2,3,4,5$.)

La traumatología ocular se puede dividir según su mecanismo de producción por agentes mecánicos (los traumatismos), por agentes físicos (quemaduras) y por agentes químicos (causticación). $(6,7)$ Siendo clasificado cada uno según tabla I.

Tabla I. Clasificación traumatología ocular.

\begin{tabular}{|l|l|}
\hline TRAUMATISMOS & Contusión, lesión incisa o traumatismo perforante. \\
\hline CUERPOS EXTRAÑOS & Superficiales o intraoculares \\
\hline QUEMADURAS & Producidas por : frío, calor, electricidad o radiación. \\
\hline CAUSTICACIONES & Producidas por: ácidos ,álcalis, disolventes o detergentes \\
\hline
\end{tabular}

Los traumatismos se dividen además según la parte a la que afecten en traumas de anejos (párpado, órbita, aparato lagrimal), polo anterior (conjuntiva, córnea, iris y cristalino) o segmento posterior.

Los cuerpos extraños alojados en órbita o globo ocular son una de las patologías urgentes más frecuentes. Serán superficiales si están sobre la conjuntiva y córnea, y profundos cuando se hallan en cristalino o segmento posterior. Esto dependerá del peso molecular, la forma y la velocidad con la que son proyectados.

Las quemaduras pueden ser ocasionadas por frío (exposición a bajas temperaturas o sustancias que al evaporarse producen congelación como gas nitrógeno), calor (llamas de las calderas, metales o cristales fundidos usados por los plateros y escaldaduras por aceite o alquitrán), electricidad (descargas de alta tensión) y radiaciones ionizantes o no ionizantes (Radiación ultravioleta como en la soldadura por arco voltaico o exposición prolongada al sol sin protección ocular).

Las causticaciones son lesiones químicas producidas principalmente por ácidos, álcalis, disolventes o detergentes. La acción de los agentes químicos depende de su concentración y del tiempo de contacto con los tejidos oculares. Los ácidos (sulfúrico de las baterías de los coches, el clorhídrico como el salfumán y el fluorhídrico usado para pulimento y grabado de vidrios, industria electrónica y fabricación de bombillas esmeriladas) provocan la coagulación de las proteínas celulares, y los álcalis o bases (amoníaco, lejías, sosa cáustica, hidróxido magnésico de las bengalas, cal de pinturas y construcción) provocan su disolución, por lo que penetran en profundidad causando mayor daño.

Las quemaduras y causticaciones se dividen según su pronóstico tal y como se ve en la tabla II

Tabla II. Quemaduras y causticaciones según su pronóstico.

\begin{tabular}{|l|l|}
\hline LEVES & Erosión epitelial sin necrosis. \\
\hline MODERADAS & Necrosis isquémica leve. \\
\hline GRAVE & Necrosis isquémica que suele evolucionar a cicatrices corneales. \\
\hline
\end{tabular}


La clasificación de urgencia de las lesiones es vital en cuanto al tratamiento, dividiéndose en: $(7,8)$

1.- Extrema urgencia: debe instaurarse tratamiento en minutos en el lugar de trabajo. Se aplica en causticaciones córneo-conjuntivales. Se trata con irrigación inmediata y prolongada con suero salino.

2.- Urgencia: puede demorarse horas. Heridas perforantes y cuerpos extraños. Los cuerpos extraños se tratarán en primer lugar irrigando a presión, si no cede la sintomatología, se aplicará anestésico para eliminar si es posible con torunda de algodón estéril y sino se intentará con aguja de 20G. Cuidado con los cuerpos metálicos por el halo de óxido que dejan alrededor. Para finalizar se prescribirá antibiótico local y se ocluirá durante 24-48 horas.

3.- No urgentes: hematomas palpebral y equimosis subconjuntival.

La misión de los equipos de protección individual (EPIs) oculares es proteger ojos y cara de la proyección de partículas sólidas, radiaciones nocivas, líquidos cáusticos y corrosivos. Basándonos en la clasificación del RD 1407/92 (11), se englobarían entre EPIs de categoría I riesgo mínimo y efecto gradual y de categoría II ya que no protegen de un peligro mortal. Los requisitos que deben cumplir se pueden resumir diciendo que deben: ser ergonómicos, ofrecer un grado de protección adecuado al riesgo, portar el marcado CE y estar acompañados de folleto explicativo en el idioma del usuario. (9)

\section{Se dividen en dos tipos:}

1.- Gafas de protección: el protector es sólo para los ojos. Pueden ser de diversos tipos: universales(montura con patillas o de montura integral en la que encierran de manera estanca la región orbital en contacto con el rostro), copas, cazoletas y máscaras.

2.- Pantallas de protección: protegen los ojos y otras zonas de cara o cabeza. Se tienen los siguientes tipos de pantallas de protección (norma EN 165: 1995):

Facial (cubre la totalidad o parte del rostro), que puede ser de mano o montada (llevada directamente sobre la cabeza mediante un arnés de cabeza, o conjuntamente con un casco de protección).

Integral, además de los ojos, cubren cara, garganta y cuello, pudiendo ser llevados sobre la cabe- za bien directamente mediante un arnés de cabeza o con un casco protector. (10)

También son obligatorias las marcas identificativas del grado de protección para el caso de oculares filtrantes. Número de código $(2=$ ultravioleta(UV) altera el reconocimiento de los colores; $3=\mathrm{UV}$ que permite un buen reconocimiento del color; $4=$ infrarrojo(IR); 5 = filtro solar sin requisitos para IR; $6=$ filtro solar con requisitos para IR) y el grado de protección que indica el "oscurecimiento" del filtro, dando una idea de la cantidad de luz visible que permite pasar.

Además pueden aparecer una serie de marcas recogidas en las normas armonizadas europeas (EN 166): identificación del fabricante, clase óptica (siempre clase 1 para los cubrefiltros), la resistencia $(\sin$ símbolo $=$ resistencia mecánica mínima, $\mathrm{S}=$ resistencia mecánica incrementada, $\mathrm{F}=$ resistencia al impacto de baja energía, $\mathrm{B}=$ resistencia al impacto de media energía, $\mathrm{A}=$ resistencia al impacto de alta energía), la resistencia a metales fundidos $n^{\circ} 9$, antiempañamiento con la letra $\mathrm{N}$, resistencia a rayado con la letra $\mathrm{K}$, campo de uso (sin símbolo: uso básico, 3: Líquidos, 4: Partículas de polvo gruesas, 5: Gas y partículas de polvo finas, 8: Arco eléctrico de cortocircuito, 9: Metal fundido y sólidos calientes).

Entre las obligaciones del empresario, se encuentran el proporcionar los equipos de protección individual gratuitamente, velar por su uso y mantenimiento, distribuirlos de manera individual, ofreciendo la correcta formación en su uso y facilitando la información a los usuarios. (11)

Las gafas de protección, pantallas o pantallas faciales, pueden ser necesarias en las siguientes actividades: Trabajo de soldadura, esmerilado o pulido y corte. Trabajos de perforación y burilado. Talla y tratamiento de piedras. Manipulación o utilización de pistolas grapadoras. Utilización de máquinas que al funcionar levanten virutas en la transformación de materiales que produzcan virutas cortas. Trabajos de estampado. Recogida y fragmentación de vidrio, cerámica, etc. Trabajo con chorro proyector de abrasivos granulosos. Manipulación o utilización de productos ácidos y alcalinos, desinfectantes y detergentes corrosivos. Manipulación o utilización de dispositivos con chorro líquido. Trabajos con masas en fusión y permanencia cerca de ellas. Actividades en un entorno de calor radiante. Trabajos con láser. Trabajos eléctricos en tensión. (5) 
Los trabajadores expuestos a riesgos biológicos, también requieren de los equipos de protección oculares. (12)

Por otra parte, se establece que los contratistas y subcontratistas están obligados a aplicar los principios de la acción preventiva, aplicando y haciendo cumplir a su personal lo establecido en el plan de seguridad y salud (10). Para que puedan hacer cumplir a sus trabajadores, tienen la posibilidad de ejercer la función sancionadora que le otorga el artículo 58.1 del RD Legislativo 1/1995 "Estatuto de los Trabajadores", por el cual los trabajadores podrán ser sancionados por la dirección de la empresa, en relación con el artículo 29 de la Ley de Prevención de Riesgos Laborales.

\section{OBJETIVO}

El objetivo de nuestro estudio es conocer la prevalencia de esta patología en nuestro medio, analizando su etiología, y proponer medidas preventivas para disminuir el número de casos, la gravedad de las lesiones y mejorar su pronóstico.

\section{MATERIAL Y MÉTODOS}

Realizamos un estudio descriptivo trasversal en los accidentes laborales que acuden al servicio de urgencias de oftalmología del Hospital Clínico San Carlos durante un período de treinta días (Abril 2006) en los que se atendió un total de 963 pacientes . El estudio incluye cuarenta y un trabajadores con accidente laboral oftalmológico. de:

Se elabora un cuestionario específico que consta

1.- Variables sociodemográficas: edad, sexo, profesión y sector profesional al que pertenecen atendiendo a la Clasificación Internacional Uniforme de Ocupaciones (CIUO-88).

2.- Variables específicas: equipos de protección ocular (dispensación y uso), reincidencia de lesiones, diagnóstico y asistencia en el lugar de trabajo.

Dicho cuestionario fue entregado y adiestrado en su cumplimentación al personal médico de guardia de oftalmología, rellenándose en caso de accidente laboral.

Analizamos todos los datos obtenidos mediante programa SPSS.

\section{RESULTADOS}

Del total de 963 atendidos en el servicio de urgencias oftalmológicas del hospital, un $16.6 \%$ corresponden a accidentes oculares. Siendo un 25.6 $\%$ de los mismos de origen laboral.

Durante el periodo de estudio la edad media de los trabajadores que acudieron a urgencias por un accidente laboral oftalmológico fue de 35 años, con un mínimo de 18 y un máximo de 63 años. Se obtiene respecto al sexo un $87 \%$ varones, y el $13 \%$ restante mujeres.

La distribución por sector profesional de la muestra está reflejada en la figura I. El sector más prevalente es el de la construcción, con un $85.4 \%$ de los trabajadores. Está seguido por el grupo de trabajadores no cualificados con un $4.9 \%$, y posteriormente los sectores científico-intelectual y servicios con un $2.4 \%$ respectivamente. En un $4.9 \%$ de los casos el sector profesional no pudo recogerse.

Figura 1. Sector profesional

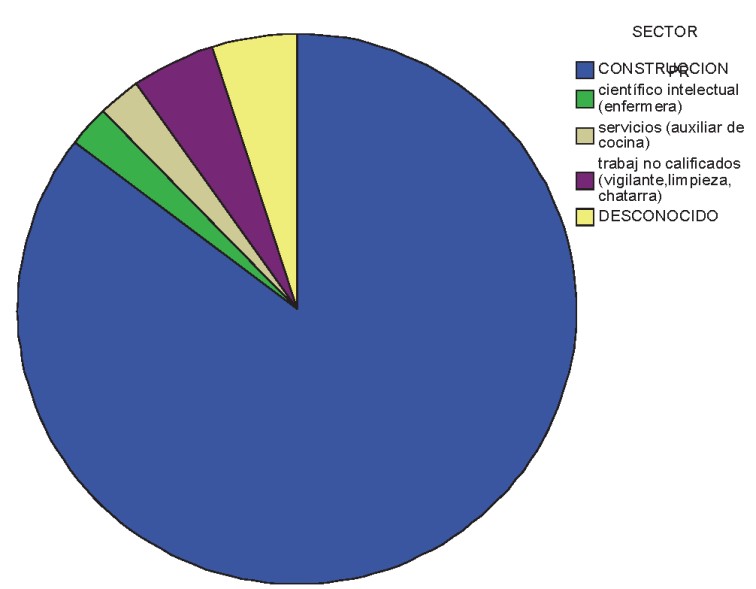

Respecto a la profesión, el $46.3 \%$ son peones de la construcción, el $19.5 \%$ soldadores, el 9.8\% mecánicos, el $4.9 \%$ electricistas y finalmente con un $2.4 \%$ cada uno tenemos a los cerrajeros, fontaneros, chatarreros, vigilantes de seguridad, enfermeros, trabajadores de limpieza, trabajadores de cocina y operarios de mantenimiento. Tomando como referencia el sector de la construcción encontramos un $34 \%$ que trabajan con radial y un $20 \%$ con equipo de soldadura.

Solamente el 51.2\% de los trabajadores accidentados afirmaban tener equipo de protección ocular. Referían no poseer dicho equipo en el $26.8 \%$, y en un $22 \%$ este dato no se recogió. De entre aquellos 
que poseen el equipo de protección ocular, obtenemos que el $48 \%$ no lo utiliza. Ver figura II.

\section{Figura 2. Uso de protección ocular}

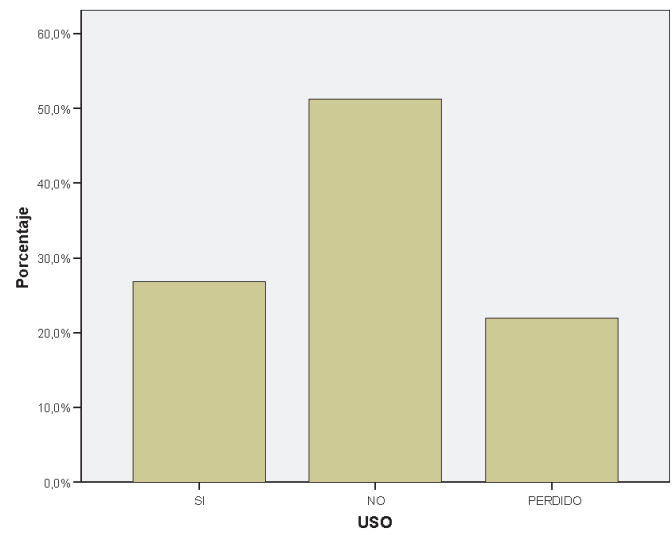

Se trataba de un accidente reiterado en un $31.7 \%$ de los casos, y era la primera vez que sufría un accidente laboral oftalmológico en un 39\%. Este dato no se obtuvo en un $29.3 \%$ de los cuestionarios.

Un 39\% de los accidentados recibieron la primera asistencia en el servicio de urgencias del hospital, mientras que en un $31.7 \%$ de los casos el paciente había recibido asistencia previa ya fuese en el lugar de trabajo o en atención primaria, y en un $29.3 \%$ no se recogió este dato.

El diagnóstico más frecuente es el de traumatismo ocular, que representa un $17.1 \%$ del total de los accidentes, en un $61 \%$ se encontró cuerpo extraño, el $17.1 \%$ de los casos sufrió quemaduras, el $2.4 \%$ causticaciones y el $2.4 \%$ infección. El diagnóstico está representado en la figura III.

\section{Figura 3. Diagnóstico}

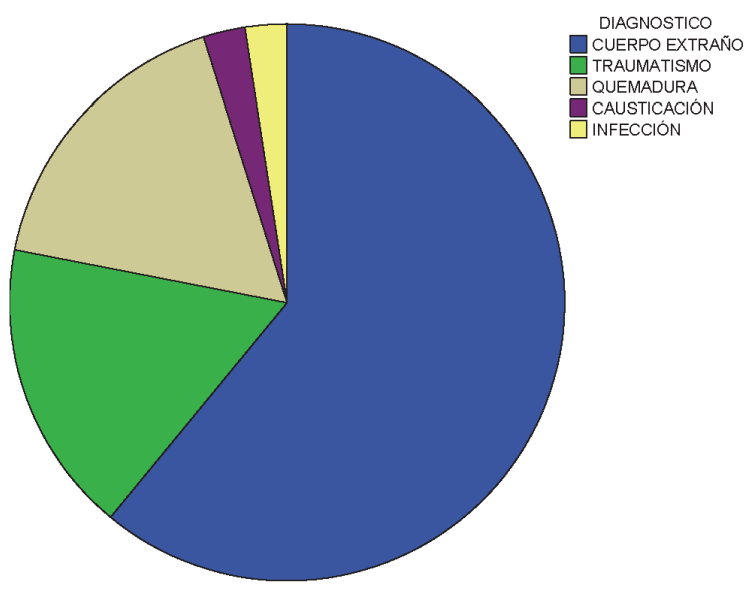

El $46 \%$ de los casos podría haber sido tratado de forma ambulatoria según criterio del oftalmólogo de guardia. Un $22 \%$ de los accidentes no eran subsidiarios únicamente de tratamiento ambulatorio, siendo recomendable la valoración por el especialista en oftalmología, y en un $32 \%$ de los casos no se completó este apartado en el cuestionario.

\section{CONCLUSIÓN}

A la vista de los resultados, encontramos que el sector más representado es el de la construcción, hombres jóvenes que trabajan con herramientas como equipo de soldadura o radial que sufren sobre todo impacto de cuerpos extraños y que en la mayoría de los casos no utiliza la protección ocular adecuada, bien porque no disponen de ella, bien porque no les resulta cómoda o por no ser consciente del riesgo al que se exponen. Vemos un porcentaje elevado de reincidencia de accidentes oculares en un mismo trabajador sin que sea, en muchos casos, razón suficiente para utilizar protección ocular. Por tanto nuestro esfuerzo debe estar encaminado a la información y formación en el uso de la protección ocular en las actividades que recoge la Ley de Prevención de Riesgos Laborales, siendo responsables el empresario en su dispensación y los trabajadores en el uso adecuado. Para esto debemos convencer a la dirección de una empresa de que cumpla con gusto esta normativa, porque además de las posibles infracciones, incluso por la vía penal debidas al incumplimiento de la ley, deberemos argumentar los riesgos a los que se exponen sus trabajadores, con las bajas que ocasionarán y por tanto las pérdidas gananciales que suponen. Al usuario habrá que convencerle igualmente de la necesidad de uso de los mismos mediante la información-formación, de los riesgos que supone el trabajo para su salud y cómo los equipos de protección ocular minimizan éstos.

Hay que tener en cuenta que los equipos de protección individual nunca sustituyen la protección colectiva, ya que no evitan el accidente, sino que minimiza sus consecuencias. Por esto mismo, aunque en el trabajo de construcción se ha visto que el usar los equipos de protección oculares minimiza las consecuencias de los accidentes, no los evita, así que estaría bien plantear la utilización de los dispositivos de seguridad como parte de la maquinaria.

En nuestra muestra según el criterio del oftalmólogo de guardia, se hubieran podido tratar de manera ambulatoria un $46.3 \%$ de los casos, por lo que el médico del trabajo debe estar entrenado para ser capaz de realizar el diagnóstico y tratamiento de 
estas patologías y actuar en el lugar de trabajo, ya que el pronóstico de las lesiones depende en gran parte del tratamiento precoz. Para ello realizaremos protocolos de actuación en caso de accidente ocular, informando al trabajador de su existencia e insistiendo en el uso de la protección ocular en caso de reincidencia.

\section{BIBLIOGRAFÍA}

1. Fernando Gil Hernández. Tratado de Medicina del Trabajo. Masson 2005.

2. J.García-Arumí, F.Escalada."Traumatismos oculares".Capítulo 14 En: Cirugía vitreorretiniana. Indicaciones y técnicas. LXXV Ponencia Oficial de la Sociedad Española de Oftalmología (1999) Borja Corgóstegui. Consultado en Abril 2006 en: http://www.oftalmo.com/publicaciones/vitreorretiniana/capitulo14.htm

3. Vasu U, Vasnaik A, Battu RR, Kurian M, George S. "Occupational open globe injuries" Indian J. Ophthalmol 2001 [cited 2006 Nov 9];49:43-47. Consultado en Abril 2006 en: http://www.ijo.in/article.asp?issn $=0301738$; year $=2001$; volume $=49$; issue $=1 ;$ spage $=43$; epage $=47$; aulast $=$ Vasu

4. Dannenberg AL, Parver LM, Brechner RJ, Khoo L. "Penetrating eye injuries in the workplace." The National Eye Trauma System Registry. Arch Ophthalmol. 1992;110:843-48.
Para finalizar es necesario tener en cuenta las limitaciones de nuestro estudio como el tiempo de realización escaso, casos no registrados debido a la presión asistencial en el servicio de urgencias de oftalmología del Hospital Clínico San Carlos de Madrid o accidentes oftalmológicos laborales leves que no requieren asistencia.

5. Saari KM, Parvi V. Occupational eye injuries in Finland. Acta Ophthalmoly Suppl. 1984;161:17-28.

6. J. C. Pastor Jimeno Guiones de Oftalmología. Mc Graw-Hill. Interamericana 2001

7. Serek Y. Kunimoto, M.D The Wills Eye Manual. Liepincott William and Wilkins 2005

8. David J. Spalton Atlas of Clinical Ophtalmology.. Elsevier Mosby 2005

9. Ley de Prevención de Riesgos Laborales (artículos 15 y 23)

10. RD 773/97. Uso de Equipos de Protección Individual.

11. RD 1627/97 Obras de Construcción. Seguridad y salud en el trabajo.

12. RD 664/97 Riesgos Biológicos. 